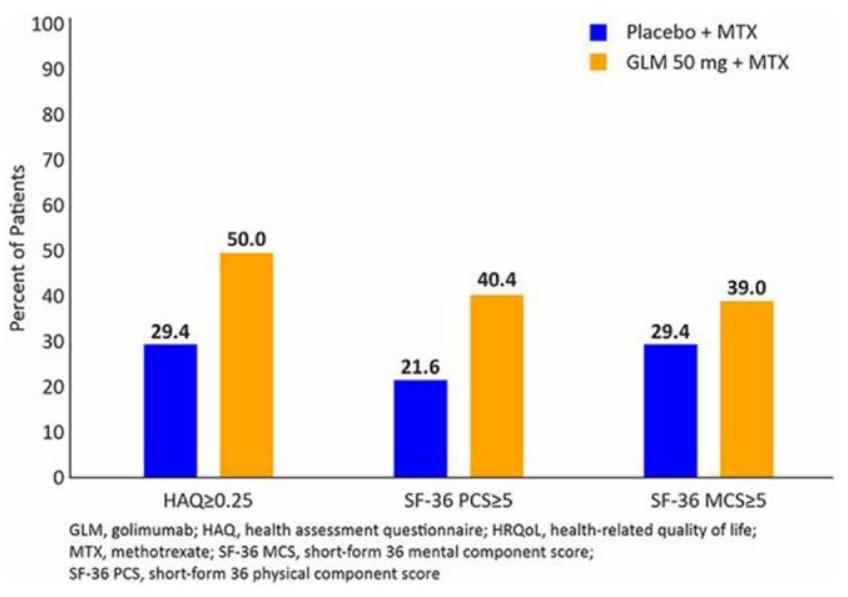

Figure 1. Proportion of Patients with Clinical Meaningful Improvements in $\mathrm{HAQ}$ and $\mathrm{HRQ}$ R: Randomised Subjects from China, Taiwan and Korea

Disclosure of Interests: Wen-Chan Tsai Consultant of: Pfizer, AbbVie, Roche, and Eli Lilly, Rong Mu: None declared, Chang-Hee Suh: None declared, Daniel Furtner Shareholder of: Johnson \& Johnson, Employee of: Johnson \& Johnson Pte. Ltd. Kim Hung Lo Shareholder of: Johnson \& Johnson, Employee of: Janssen Research \& Development, LLC, Yiying Zhou Shareholder of: Johnson \& Johnson, Employee of: Janssen Research \& Development, LLC, Elizabeth C Hsia Shareholder of: Johnson \& Johnson, Employee of: Janssen Research \& Development, LLC DOI: 10.1136/annrheumdis-2021-eular.205

\section{POS0438 IS IMAGING DETECTED JOINT INFLAMMATION HELPFUL IN EXPLAINING FATIGUE IN RHEUMATOID ARTHRITIS AT DIAGNOSIS AND DURING THE DISEASE COURSE? - A LARGE MRI STUDY}

X. Matthijssen ${ }^{1}$, F. Wouters ${ }^{1}$, N. Sidhu ${ }^{1}$, A. van der Helm - van Mil ${ }^{1,2} .{ }^{1}$ Leiden University Medical Center (LUMC), Rheumatology, Leiden, Netherlands; ${ }^{2}$ Erasmus MC, Rheumatology, Rotterdam, Netherlands

Background: Fatigue in rheumatoid arthritis (RA) is hypothesized to be caused by inflammation. Still $\sim 50 \%$ of fatigue in RA cannot be explained by the disease activity score (DAS), nor by generic or psychological factors.

Objectives: Since MRI can detect joint inflammation more sensitively than DAS, we hypothesized that residual inflammation detected by MRI could aid in explaining fatigue in RA at diagnosis and during follow-up.

Methods: 526 consecutive RA-patients were followed longitudinally. Fatigue was assessed yearly on a numerical rating scale. Hand and foot MRIs were performed at inclusion, after 12 and 24-months in 199 patients and were scored for inflammation (synovitis, tenosynovitis and osteitis combined). We studied whether RA-patients with more MRI-inflammation were more fatigued at diagnosis (linear regression), whether the 2-year course of MRI-inflammation associated with the course of fatigue (linear mixed models) and whether decrease in MRI-inflammation in year-1 associated with subsequent improvement in fatigue in year-2 (cross-lagged models). Similar analyses were done with DAS as inflammation measure.

Results: At diagnosis, higher DAS-scores were associated with more severe fatigue $(p<0.001)$. However, patients with more MRI-inflammation were not more fatigued $(\mathrm{p}=0.94)$. During 2-year follow-up, DAS decrease associated with improvement in fatigue $(\mathrm{p}<0.001)$, but MRI-inflammation decrease did not $(p=0.96)$. DAS decrease in year-1 associated with fatigue improvement in year-2 $(p=0.012)$, as did MRI-inflammation decrease $(p=0.039)$, with similar effect strength.

Conclusion: Sensitive measurements of joint inflammation did not aid in explaining fatigue in RA at diagnosis and follow-up. This supports the concept that fatigue in RA is partly uncoupled from inflammation.

Disclosure of Interests: None declared

DOI: 10.1136/annrheumdis-2021-eular.258

\section{POS0439 THE INFLUENCE OF ESCALATED PGA SCORE ON DISEASE ACTIVITY, DAILY ACTIVITY, AND QUALITY OF LIFE, AND SOLUTION FOR OPTIMAL PGA LEVEL THAT DESERVES CLINICAL REMISSION IN PATIENT WITH RHEUMATOID ARTHRITIS}

1. Yoshii'. 'Yoshii Hospital, Rheumatology and Musculoskeletal Medicine, Shimanto City, Japan
Background: Patient's global assessment (PGA) is one most difficult component as a part of disease activity index for treatment of rheumatoid arthritis (RA), that often causes an obstacle to attaining clinical remission. Moreover, PGA level affects activities in daily living

Objectives: The influence of escalated PGA score on disease activity, daily activity, and quality of life for patient with RA was investigated., and the optimal PGA level for both disease activity and daily activities was investigated from real world data.

Methods: A total of 24,075 times of monitoring for RA was performed in the institute. Monitored items included TJC, SJC, PGA, EGA, CRP, and calculated values of DAS28, CDAI, SDAI, composite index of Boolean evaluation (Boolean), pain score with visual analog scale (PS-VAS), Health Assessment Questionnaire Disability Index (HAQ-DI), and quality of life score (QOLS) calculated from Euro$\mathrm{QOL}$ questionnaire with $5^{\text {th }}$ dimensions. Each measured item was calculated as mean value according to the PGA score, which was measured at the same time. The PGA score was classified by one increment from zero to ten. The mean values of DAS28, CDAI, SDAl, remission rate of these indices and Boolean remission rate, and mean values of PS-VAS, HAQ-DI, and QOLS were statistically evaluated.

HAQ-DI below 0.5 was determined as remission (HAQ remission). Sensitivity and specificity regarding attaining $\mathrm{HAQ}$ remission according to each level of PGA score were calculated, and cutoff index (COI) was determined with receiver operating characteristic (ROC) curve. For PS-VAS, sensitivity and specificity of Boolean remission regarding each level of PS-VAS after classification divided by one increment was calculated, and comparable level (PS-VAS remission) was determined with reference of the curve. ROC was performed according to PGA level, and $\mathrm{COI}$ was determined with a same manner.

Results: Number of measures counted 10428, 3099, 3110, 2346, 998, 1773 $751,703,655,139$, and 73 for each PGA level. PGA level from 3 to 5 , and 5 to 10 were put together for number adjusting.

Mean DAS28, CDAl, and SDAI demonstrated significant increase as PGA level increased, and remission rate of the all indices including Boolean demonstrated significant decrease as PGA level increases $(\mathrm{p}<0.01 \%)$. Boolean remission rate demonstrated zero percent from two, and CDAI and SDAI remission rate demonstrated zero from five, whereas DAS remission rate showed gradual decrease then zero percent was not shown in any level. Mean value of PS-VAS and HAQ-DI score demonstrated also significant decrease as PGA level increases, and QOLS demonstrated significant decrease as PGA level increases $(p<0.01 \%)$. Increase of HAQ-DI score and decline of QOLS demonstrated more steep from PGA level 3 , whereas no significant difference demonstrated from zero to one. $\mathrm{HAQ}$ remission counted 15,703 , whereas no $\mathrm{HAQ}$ remission counted 8,335. Using ROC, COI of the PGA level was 2.0, whereas sensitivity and specificity were $63.4 \%$ and $66.3 \%$, respectively. The estimated PS-VAS remission level was $10 \mathrm{~mm}$. Optimal PGA level for PS-VAS remission was set as 1.0 , and sensitivity and specificity regarding PS-VAS remission were $87.1 \%$ and $71.3 \%$, respectively. Conclusion: Increase of PGA affects daily activities and quality of life. The evident level that increases deterioration risk significantly was supposed to be from 3. Optimal level of PGA score for attaining the PS-VAS remission was 1.0, whereas the optimal PGA level for HAQ-DI remission is 1.0, despite sensitivity and specificity for the HAQ remission were lower than these for the PS-VAS remission.

Disclosure of Interests: None declared

DOI: 10.1136/annrheumdis-2021-eular.285

\section{POS0440 REDUCTION IN MONOCYTE COUNT PREDICTS SUSTAINED REMISSION IN RHEUMATOID ARTHRITIS PATIENTS TREATED WITH ANTI-TNF THERAPY}

R. Amarnani ${ }^{1}$, M. Mainuddin ${ }^{2}$, M. Shipa ${ }^{3}$, S. A. Yeoh ${ }^{3}$, M. Ehrenstein ${ }^{1,3}$ ${ }^{1}$ University College Hospital, Rheumatology, London, United Kingdom; ${ }^{2}$ North Middlesex University Hospital, Rheumatology, London, United Kingdom; ${ }^{3}$ University College London, Centre for Rheumatology, Division of Medicine, London, United Kingdom

Background: Sustained remission is the ultimate goal in the management of rheumatoid arthritis (RA) but is infrequently achieved. After conventional therapy, $\mathrm{TNFi}$ (tumour necrosis factor inhibitor) has a strong track record for achieving remission. Existing studies suggest variable effect of RA and TNFi on various cellular components of the full blood count (FBC), but their relationship with loss of remission (LOR) is unclear.

Objectives: To investigate whether cellular changes in the FBC can predic LOR (remission defined as DAS28-ESR $\leq 2.6$ ) in patients with RA receiving TNFi, adjusted using key clinical factors.

Methods: Real-world clinical and routine laboratory data were analysed from two independent cohorts of adult RA patients, who were started on their first TNF (from 2009 to December 2019), and went into remission. Data was extracted in October 2020. A linear mixed model was used to investigate longitudinal changes of different components of FBC and CRP, stratified by LOR, and grouped by 
years of follow-up. Pairwise post-hoc comparisons were performed by Bonferroni correction. Area Under the Receiver Operating Characteristics (AUROC) of cellular changes in FBC components to predict LOR at different time points were calculated. Latent class mixed models were used to investigate trajectories of change in cellular components of the FBC from baseline to one year. Further survival analysis to predict LOR of TNFi was done by using the latent class model and adjusted by clinical parameters, demographics and concomitant treatment. Results: 92 and 43 biologic-naïve RA patients who attained remission were included from cohorts 1 and 2 respectively. 73 (54\%) were treated with adalimumab and $62(46 \%)$ with etanercept. $87(64 \%)$ of patients lost remission over a 10 -year period with median time to LOR of 3.3 years $(95 \% \mathrm{Cl} 2.6-3.7)$.

Among the cellular components of FBC, monocyte count changes in the first year after initiation of TNFi fell significantly in those who maintained remission over the five years of follow-up, compared to those who lost remission $[p<0.001$ for all years evaluated] (Figure 1). AUROC of monocyte count change at 6 months and 12 months, to predict LOR from year 1 to year 5 (after TNFi initiation) and after 5 years, ranged between 0.70-0.90. Three latent classes were identified based on monocyte count changes over time: 1-decrease $(\mathrm{N}=41)$, 2-increase $(\mathrm{N}=7)$ and 3- no change $(\mathrm{N}=87)$. Among these latent classes, patients in class 1 , sustained remission for longer (unadjusted log-rank $p=0.001$ ) compared to the other 2 classes. Monocyte latent classes as a predictor of LOR was further tested by cox-regression, where variables (anti-citrullinated protein antibody/ACPA, concomitant methotrexate, baseline DAS28-ESR) were selected by elastic net regularisation and further adjusted by use of steroid, age and gender. Class 2 and Class 3 monocyte latent classes showed a hazard ratio (HR) of $3.15(95 \% \mathrm{Cl}$ 1.02-9.74, $p=0.046, \mathrm{n}$. of event $=6 / 7$ ) and $5.48(95 \% \mathrm{Cl} 2.6-11.57, p<0.001, \mathrm{n}$. of event $=73 / 87$ ) respectively compared to Class 1 . ACPA positivity also increased the risk of LOR with $\mathrm{HR}$ of 2.13 and $95 \% \mathrm{Cl} 1.04-4.37, p=0.04$ (n. of event $=$ $78 / 117$ ) compared to seronegative patients ( $n$. of event $=9 / 18$ ).

Conclusion: In seropositive RA patients treated with $\mathrm{TNFi}$, a reduction of total monocyte count in the first year was associated with maintenance of remission in subsequent years. Further studies are required to determine whether these effects are specific to TNFi or reflect sustained remission in RA irrespective of therapy.

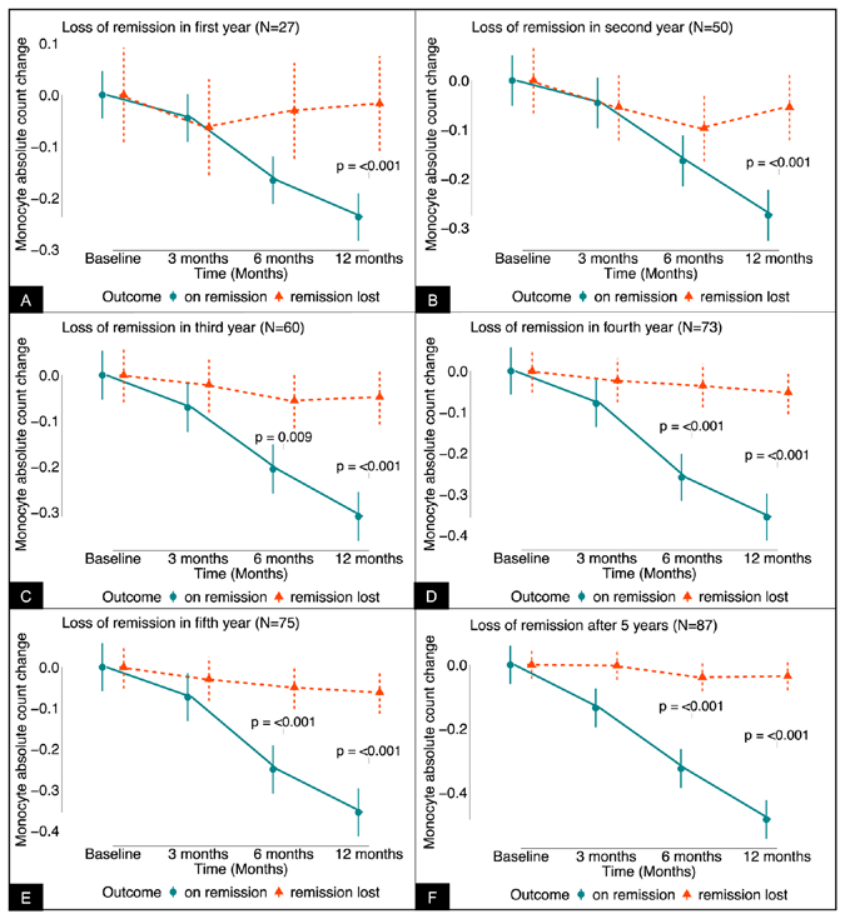

Figure 1 (A-F). Mean change (with 95\% confidence interval) in absolute count of monocyte after initiation of tumour necrosis factor inhibitor grouped by years of follow-up and stratified by loss of remission. $\mathrm{N}=$ number indicate cumulative number of patients who lost remission by the end of each year.

Acknowledgements: Muhammad Shipa is funded by Versus Arthritis. Su-Ann Yeoh is funded by the Royal College of Physicians, Rosetrees Trust, NIHR University College London Hospitals Biomedical Research Centre, UCLH Charities, and Versus Arthritis. Michael Ehrenstein is supported (in part) by the University College London Hospital Biomedical Research Centre. None of the funding bodies have been involved in preparation of this manuscript.

Disclosure of Interests: None declared

DOI: 10.1136/annrheumdis-2021-eular.312

\section{POS0441 DEVELOPMENT OF RHEUMATOID ARTHRITIS AMONG ANTI-CITRULLINATED PROTEIN ANTIBODIES POSITIVE ASYMPTOMATIC INDIVIDUALS: A PROSPECTIVE OBSERVATIONAL STUDY}

S. Mizuki ${ }^{1}$, K. Horie ${ }^{1}$, K. Imabayashi ${ }^{1}$, K. Mishima ${ }^{2}$, K. Oryoji ${ }^{1}{ }^{1}$ Matsuyama Red Cross Hospital, The Center for Rheumatic Diseases, Matsuyama, Japan; ${ }^{1}$ Matsuyama Red Cross Hospital, The Center for Rheumatic Diseases, Matsuyama, Japan

Background: In the idividuals with genetic and enviromental risk factors, immune events at mucosal surfaces occur and may precede systemic autoimmunity. Anti-citrullinated protein antibodies (ACPA) are present in the serum for an average of 3-5 years prior to the onset of rheumatoid arthritis (RA) during an asymptomatic period. In ACPA-positivite individuals, the additional presence of RA-related risk factors appears to add significant power for the development of RA. To date, there have been few reports in which clinical courses of ACPA-positive asymptomatic individuals were investigated prospectively.

Objectives: To observe the clinical time course of ACPA-positive healthy population for the development of RA.

Methods: Healthy volunteers without joint pain or stiffness, who attended the comprehensive health screening of our hospital, were enrolled in this prospective observational study. The serum ACPA levels were quantified by Ig-G anti-cyclic citrullinated peptide enzyme-linked immunosorbent assay with levels $>4.4 \mathrm{U} / \mathrm{mL}$ considered positive. ACPA-positive subjects were followed by rheumatologists of our department clinically or a questionnaire sent by mail for screening to detect arthritis.

Results: 5,971 healthy individuals without joint symptons were included. Ninty-two (1.5\%) were positive for ACPA. Of these, 19 (20.7\%) developed RA and two were suspected as RA by mail questionnaire. Their average age were 58 -years, and women were $68 \%$. The average duration between the date of serum sampling and diagnosis was 10.7 months. ACPA-positive individuals who developed to RA had higher serum ACPA and Ig-M rheumatoid factor levels than ACPA-positive individuals who did not ( $P$ value by Mann-Whitney $U$ test: 0.002 , 0.005 , respectively).

Conclusion: Among ACPA-positive asymptomatic individuals, 20\% developed $\mathrm{RA}$. The higher titer of ACPA and Ig-M rheumatoid factor levels are risk factors for devoloping RA.

Disclosure of Interests: None declared

DOI: 10.1136/annrheumdis-2021-eular.344

\begin{tabular}{|l|l|}
\hline POS0442 & RELATIONSHIP BETWEEN PARAOXONASE-1 \\
GENOTYPE, ACTIVITY AND MAJOR ADVERSE \\
CARDIOVASCULAR EVENTS IN PATIENTS WITH \\
RHEUMATOID ARTHRITIS RECEIVING TOFACITINIB
\end{tabular}

C. Charles-Schoeman ${ }^{1}$, C. Hyde ${ }^{2}$, S. Guan ${ }^{3}$, N. Parikh ${ }^{1}$, J. Wang ${ }^{1}$

A. Shahbazian ${ }^{1}$, L. Stockert ${ }^{4}$, J. Andrews ${ }^{4} .{ }^{1}$ University of California, Department of Medicine, Los Angeles, CA, United States of America; ${ }^{2}$ Pfizer Inc, Inflammation and Immunology, Groton, CT, United States of America; ${ }^{3}$ Pfizer Inc, Inflammation and Immunology, Cambridge, MA, United States of America ${ }^{4}$ Pfizer Inc, Inflammation and Immunology, Collegeville, PA, United States of America

Background: Paraoxonase-1 (PON1) is a high-density lipoprotein (HDL)-associated enzyme with paraoxonase, lactonase and arylesterase activities. ${ }^{1}$ PON1 contributes to the antioxidant properties of $\mathrm{HDL}$, and is being investigated for its atheroprotective properties. ${ }^{1}$ Patients (pts) with rheumatoid arthritis (RA) who are homozygous for the RR genotype of the Q192R gene polymorphism on PON1 (rs662) have increased paraoxonase activity, and lower risk of carotid plaques, vs those with $Q Q$ or $Q R$ genotypes. ${ }^{2}$ Tofacitinib is an oral Janus kinase inhibitor for the treatment of RA.

Objectives: To investigate the relationship between PON1 genotype/activity and risk of major adverse cardiovascular events (MACE) in the tofacitinib RA clinical programme.

Methods: In this post hoc analysis, data were pooled from pts enrolled in nine Phase 2/3 studies of tofacitinib in RA. Enzyme activities in pt plasma samples were measured at individual study baseline $(B L)$ and at follow-up visits using three substrates: paraoxon (paraoxonase activity), dihydrocoumarin (lactonase activity) and phenylacetate (arylesterase activity). The effect of the PON1 Q192R genotype ( $\mathrm{QQ}, \mathrm{QR}$ or $\mathrm{RR}$ ) on $\mathrm{BL}$ paraoxonase/lactonase/arylesterase activity was assessed using linear regression for each study, with age and sex as covariates, and then fixed-effect meta-analysis assessed effects across studies. The risk of MACE by enzyme activity was determined using Cox proportional hazards regression stratified by clinical studies. Univariate regression against $B L$ enzyme activity and other risk factors, as well as both minimally and fully adjusted multivariable regressions against time-varying enzyme activity, are presented.

Results: The analysis included 1969 pts with RA who received $\geq 1$ dose of tofacitinib and had PON1 activity measures available at BL; 39 pts had $\geq 1$ 